Bull. Korean Math. Soc. 46 (2009), No. 6, pp. 1135-1140

DOI 10.4134/BKMS.2009.46.6.1135

\title{
PRODUCTS OF DIFFERENTIATION AND COMPOSITION ON BLOCH SPACES
}

\author{
SHÛICHI OHNO
}

\begin{abstract}
We will consider the questions of when the products of composition and differentiation are bounded and compact on Bloch and little Bloch spaces.
\end{abstract}

\section{Introduction}

Let $\mathbb{U}$ denote the open unit disk in the complex plane and $\mathcal{H}(\mathbb{U})$ be the vector space of all analytic functions on $\mathbb{U}$. For an analytic self-map $\varphi$ of $\mathbb{U}$, we define the composition operator $C_{\varphi}$ on $\mathcal{H}(\mathbb{U})$ by

$$
C_{\varphi} f(z)=f(\varphi(z)) \text { for } \quad z \in \mathbb{U} \text { and } f \in \mathcal{H}(\mathbb{U}) .
$$

The Littlewood Subordination Principle shows that the restriction of $C_{\varphi}$ to the Hardy or weighted Bergman spaces is always bounded. Composition of functions is one of the most common and fundamental operations in mathematics. Thus many aspects of composition operators have been investigated on various analytic function spaces. One of such problems is the study of weighted composition operators, which appear in the theory of isometries on the spaces of analytic functions. From a different perspective, this problem would be yielded for the consideration of the operator range of the composition operators. Explicitly, it is asked how large enough the range of the composition operator is for the multiplication operators to be well acting there. We refer to the books [2] and [7] for the theory of composition operators.

Hibschweiler and Portnoy [4] considered the behavior of the differentiation on the range of the composition operator on the Hardy or weighted Bergman spaces. Let $D$ be the differentiation operator and $C_{\varphi}$ the operator of composition with an analytic self-map $\varphi$ of $\mathbb{U}$. Then define the products of these operators by

$$
D C_{\varphi} f(z)=\left(C_{\varphi} f\right)^{\prime}(z)=f^{\prime}(\varphi(z)) \varphi^{\prime}(z)
$$

Received September 4, 2008.

2000 Mathematics Subject Classification. Primary 47B38; Secondary 30H05.

Key words and phrases. differentiation, composition operators, Bloch spaces.

The author is partially supported by Grant-in-Aid for Scientific Research (No.20540185), Japan Society for the Promotion of Science. 
and

$$
C_{\varphi} D f(z)=\left(C_{\varphi} f^{\prime}\right)(z)=f^{\prime}(\varphi(z))
$$

for $z \in \mathbb{U}$ and $f \in \mathcal{H}(\mathbb{U})$.

Generally $D$ is unbounded on analytic function spaces. Hibschweiler and Portnoy [4] characterized the boundedness and the compactness of $D C_{\varphi}$ and $C_{\varphi} D$ between weighted Bergman spaces using the Carleson-type measures. But such weighted Bergman spaces would not include the Hardy space case in the characterization of boundedness and compactness of $C_{\varphi} D$. The author [5] studied this remnant.

In this note we consider the products $D C_{\varphi}$ and $C_{\varphi} D$ on the Bloch spaces. The Bloch space $\mathcal{B}$ is the space of all analytic functions $f$ on $\mathbb{U}$ for which

$$
\sup _{z \in \mathbb{U}}\left(1-|z|^{2}\right)\left|f^{\prime}(z)\right|<\infty
$$

and the little Bloch space $\mathcal{B}_{o}$ consists of all $f \in \mathcal{B}$ for which

$$
\lim _{|z| \rightarrow 1}\left(1-|z|^{2}\right) f^{\prime}(z)=0 \text {. }
$$

Endowed with the norm

$$
\|f\|_{\mathcal{B}}=|f(0)|+\sup _{z \in \mathbb{U}}\left(1-|z|^{2}\right)\left|f^{\prime}(z)\right|,
$$

the Bloch space $\mathcal{B}$ becomes a Banach space and $\mathcal{B}_{o}$ is a closed subspace of $\mathcal{B}$. Moreover a higher derivative analogue of Bloch functions also is given: Suppose that $f$ is analytic on $\mathbb{U}$ and $n \geq 2$. Then $f \in \mathcal{B}$ if and only if $\left(1-|z|^{2}\right)^{n}\left|f^{(n)}(z)\right|$ is bounded on $\mathbb{U}$. It is well known that a Bloch function can grow at most as fast as $\log (1 /(1-|z|))$, that is, for $f \in \mathcal{B}$ with $\sup _{z \in \mathbb{U}}\left(1-|z|^{2}\right)\left|f^{\prime}(z)\right| \leq 1$,

$$
|f(z)-f(0)| \leq \frac{1}{2} \log \frac{2}{1-|z|} .
$$

See [9, Chap. 5] for more information on Bloch spaces.

In the next section we characterize the boundedness and the compactness of $D C_{\varphi}$ on the Bloch and little Bloch spaces. In Section 3, we will consider the opposite form $C_{\varphi} D$ on the Bloch and little Bloch spaces. Note that $D$ is bounded neither on $\mathcal{B}$ nor on $\mathcal{B}_{o}$ but $C_{\varphi}$ is always bounded on $\mathcal{B}$ by the Schwarz-Pick inequality. Our theorems give interesting relations to the analytic Zygmund class and the generalized Schwarz-Pick inequality.

\section{The product $D C_{\varphi}$ on Bloch spaces}

In this section we will show that the boundedness and compactness of $D C_{\varphi}$ coincide using the compactness of composition operators on the analytic Lipschitz space.

To characterize the compactness, we will need the following result, whose proof is an easy modification of that of Proposition 3.11 in [2]. 
Proposition 2.1. Let $\varphi$ be an analytic self-map of $\mathbb{U}$. Then $D C_{\varphi}\left(\right.$ or $\left.C_{\varphi} D\right)$ is compact on $\mathcal{B}$ if and only if whenever $\left\{f_{n}\right\}$ is bounded in $\mathcal{B}$ and $f_{n} \rightarrow$ 0 uniformly on compact subsets of $\mathbb{U}$, then $D C_{\varphi} f_{n} \rightarrow 0\left(\right.$ or $C_{\varphi} D f_{n} \rightarrow 0$, respectively) in $\mathcal{B}$.

We will consider as follows. For $f \in \mathcal{B}$,

$$
D C_{\varphi} f(z)=f^{\prime}(\varphi(z)) \varphi^{\prime}(z), \quad z \in \mathbb{U} .
$$

Recall that a function $f \in \mathcal{H}(\mathbb{U})$ is said to belong to the Bloch-type space $\mathcal{B}^{2}$ if

$$
\sup _{z \in \mathbb{U}}\left(1-|z|^{2}\right)^{2}\left|f^{\prime}(z)\right|<\infty \text {. }
$$

We here notice that the boundedness of $D C_{\varphi}$ from $\mathcal{B}$ to $\mathcal{B}$ is equivalent to the boundedness of the weighted composition operator $\varphi^{\prime} C_{\varphi}$ from $\mathcal{B}^{2}$ to $\mathcal{B}$. So using the results in [6], we obtain the following theorems.

Theorem 2.2. Let $\varphi$ be an analytic self-map of $\mathbb{U}$. Then the following are equivalent:

(i) $D C_{\varphi}: \mathcal{B} \rightarrow \mathcal{B}$ is bounded.

(ii) $D C_{\varphi}: \mathcal{B} \rightarrow \mathcal{B}$ is compact.

(iii) $\varphi^{\prime} \in \mathcal{B}$ and $\|\varphi\|_{\infty}<1$.

Proof. We firstly show that (i) implies (iii). It is trivial that $D C_{\varphi} z=\varphi^{\prime} \in \mathcal{B}$.

As the argument above, we obtain the following conditions which are equivalent to the boundedness of the weighted composition operator $\varphi^{\prime} C_{\varphi}$ from $\mathcal{B}^{2}$ to $\mathcal{B}$, using the results in $[6]$ :

$$
\sup _{z \in \mathbb{U}} \frac{1-|z|^{2}}{1-|\varphi(z)|^{2}}\left|\varphi^{\prime \prime}(z)\right|<\infty
$$

and

$$
\sup _{z \in \mathbb{U}} \frac{1-|z|^{2}}{\left(1-|\varphi(z)|^{2}\right)^{2}}\left|\varphi^{\prime}(z)\right|^{2}<\infty .
$$

For example, we also get the condition (2.2), using test functions

$$
f(z)=\frac{\left(1-|\varphi(\lambda)|^{2}\right)^{2}}{2(1-\overline{\varphi(\lambda)} z)^{2}}-\frac{1-|\varphi(\lambda)|^{2}}{1-\overline{\varphi(\lambda)} z}
$$

for $\lambda \in \mathbb{U}$.

Here we recall that the analytic Lipschitz space $\mathcal{B}^{1 / 2}$ consists of all analytic functions $f$ on $\mathbb{U}$ satisfying the condition

$$
\sup _{z \in \mathbb{U}}\left(1-|z|^{2}\right)^{1 / 2}\left|f^{\prime}(z)\right|<\infty .
$$

Thus, by (2.1) and (2.2), $\varphi$ is in the analytic Lipschitz space $\mathcal{B}^{1 / 2}$ and so

$$
\sup _{z \in \mathbb{U}}\left(\left(1-|z|^{2}\right) /\left(1-|\varphi(z)|^{2}\right)\right)^{1 / 2}\left|\varphi^{\prime}(z)\right|<\infty .
$$


So we can easily obtain that $C_{\varphi}: \mathcal{B}^{1 / 2} \rightarrow \mathcal{B}^{1 / 2}$ is bounded. (This is well known. See [6, Corollary 2.4], for example.)

Moreover, if $|\varphi(\lambda)| \rightarrow 1$,

$$
\lim _{|\varphi(\lambda)| \rightarrow 1} \frac{\left(1-|\lambda|^{2}\right)\left|\varphi^{\prime}(\lambda)\right|^{2}}{1-|\varphi(\lambda)|^{2}}=0
$$

by $(2.2)$.

If $|\lambda| \rightarrow 1$ and $|\varphi(\lambda)| \rightarrow r<1$

$$
\frac{\left(1-|\lambda|^{2}\right)\left|\varphi^{\prime}(\lambda)\right|^{2}}{1-|\varphi(\lambda)|^{2}} \leq \frac{1-|\lambda|^{2}}{1-|\varphi(\lambda)|^{2}}\left(\|\varphi\|_{\mathcal{B}} \frac{1}{2} \log \frac{2}{1-|\lambda|}\right)^{2} \rightarrow 0 .
$$

So from (2.2) we obtain the condition;

$$
\lim _{|\lambda| \rightarrow 1} \frac{1-|\lambda|^{2}}{1-|\varphi(\lambda)|^{2}}\left|\varphi^{\prime}(\lambda)\right|^{2}=\lim _{|\lambda| \rightarrow 1}\left(\frac{1-|\lambda|^{2}}{1-|\varphi(\lambda)|^{2}}\right)^{1 / 2}\left|\varphi^{\prime}(\lambda)\right|=0 .
$$

This condition is equivalent to the fact that $C_{\varphi}: \mathcal{B}^{1 / 2} \rightarrow \mathcal{B}^{1 / 2}$ is compact. (This also is well known. Refer to [6, Corollary 3.2], for example.) Thus by [2, Theorem 4.5], we have $\|\varphi\|_{\infty}<1$. So the condition (iii) holds.

Next we will show the implication (iii) $\Rightarrow$ (ii) using Proposition 2.1. Suppose that $\left\{f_{n}\right\}$ is bounded in $\mathcal{B}$ and $f_{n} \rightarrow 0$ uniformly on compact subsets of $\mathbb{U}$ as $n \rightarrow \infty$.

By the condition (iii), $K=\left\{w \in \mathbb{U}:|w| \leq\|\varphi\|_{\infty}\right\}$ is a compact subset of $\mathbb{U}$. Then we have

$$
\begin{aligned}
& \left\|D C_{\varphi} f_{n}\right\|_{\mathcal{B}} \\
= & \left|D C_{\varphi} f_{n}(0)\right|+\sup _{z \in \mathbb{U}}\left(1-|z|^{2}\right)\left|\left(D C_{\varphi} f_{n}\right)^{\prime}(z)\right| \\
\leq & \left|D C_{\varphi} f_{n}(0)\right| \\
& +\sup _{z \in \mathbb{U}}\left(1-|z|^{2}\right)\left|f_{n}^{\prime \prime}(\varphi(z))\right|\left|\varphi^{\prime}(z)\right|^{2}+\sup _{z \in \mathbb{U}}\left(1-|z|^{2}\right)\left|f_{n}^{\prime}(\varphi(z)) \varphi^{\prime \prime}(z)\right| .
\end{aligned}
$$

The first term on the right-hand side clearly tends to zero as $n \rightarrow \infty$. The second term is bounded by

$$
\begin{aligned}
& \sup _{z \in \mathbb{U}}\left(1-|z|^{2}\right)\left|f_{n}^{\prime \prime}(\varphi(z))\right|\left|\varphi^{\prime}(z)\right|^{2} \\
\leq & \sup _{w \in K}\left|f_{n}^{\prime \prime}(w)\right| \sup _{z \in \mathbb{U}}\left(1-|z|^{2}\right)\left(\|\varphi\|_{\mathcal{B}} \frac{1}{2} \log \frac{2}{1-|\lambda|}\right)^{2} .
\end{aligned}
$$

As $\left(1-|z|^{2}\right)(\log 2 /(1-|z|))^{2}$ is bounded, the second also tends to zero as $n \rightarrow \infty$.

Furthermore the third term is bounded by

$$
\sup _{w \in K}\left|f_{n}^{\prime}(w)\right| \sup _{z \in \mathbb{U}}\left(1-|z|^{2}\right)\left|\varphi^{\prime \prime}(z)\right| \leq \sup _{w \in K}\left|f_{n}^{\prime}(w)\right|\left\|\varphi^{\prime}\right\|_{\mathcal{B}} .
$$

Thus this term also tends to zero as $n \rightarrow \infty$.

Consequently,

$$
\left\|D C_{\varphi} f_{n}\right\|_{\mathcal{B}} \rightarrow 0 \quad \text { as } \quad n \rightarrow \infty
$$


that is, $D C_{\varphi}: \mathcal{B} \rightarrow \mathcal{B}$ is compact.

The implication (ii) $\Rightarrow(\mathrm{i}$ ) is clear.

Here we add the notion of the analytic Zygmund class $\Lambda^{*}$, that is,

$$
\Lambda^{*}=\left\{f \text { is analytic on } \mathbb{U}: \sup _{z \in \mathbb{U}}\left(1-|z|^{2}\right)\left|f^{\prime \prime}(z)\right|<\infty\right\}
$$

endowed with the norm

$$
\|f\|_{\Lambda^{*}}=|f(0)|+\left|f^{\prime}(0)\right|+\sup _{z \in \mathbb{U}}\left(1-|z|^{2}\right)\left|f^{\prime \prime}(z)\right| .
$$

(Refer to [3].)

Then each condition in Theorem 2.2 furthermore is equivalent to the following condition:

(iv) $C_{\varphi}: \mathcal{B} \rightarrow \Lambda^{*}$ is bounded.

In a similar manner to Theorem 2.2 we can obtain the following theorem which describes the boundedness and the compactness of $D C_{\varphi}$ on the little Bloch space $\mathcal{B}_{o}$.

Theorem 2.3. Let $\varphi$ be an analytic self-map of $\mathbb{U}$. Then the following are equivalent:

(i) $D C_{\varphi}: \mathcal{B}_{o} \rightarrow \mathcal{B}_{O}$ is bounded.

(ii) $D C_{\varphi}: \mathcal{B}_{o} \rightarrow \mathcal{B}_{o}$ is compact.

(iii) $\varphi^{\prime} \in \mathcal{B}_{o}$ and $\|\varphi\|_{\infty}<1$.

\section{The product $C_{\varphi} D$ on Bloch spaces}

In this section we will consider the opposite form $C_{\varphi} D$. Also, using theorems in [6], we can show the following results.

Theorem 3.1. Let $\varphi$ be an analytic self-map of $\mathbb{U}$. Then the following are equivalent:

(i) $C_{\varphi} D: \mathcal{B} \rightarrow \mathcal{B}$ is bounded.

(ii) $C_{\varphi}: \mathcal{B}^{2} \rightarrow \mathcal{B}$ is bounded.

(iii)

$$
\sup _{z \in \mathbb{U}} \frac{1-|z|^{2}}{\left(1-|\varphi(z)|^{2}\right)^{2}}\left|\varphi^{\prime}(z)\right|<\infty .
$$

Analogous to Theorem 2.2, the following result characterizes the compactness of $C_{\varphi} D$ using Proposition 2.1.

Theorem 3.2. Suppose that $C_{\varphi} D: \mathcal{B} \rightarrow \mathcal{B}$ is bounded. Then the following are equivalent:

(i) $C_{\varphi} D: \mathcal{B} \rightarrow \mathcal{B}$ is compact.

(ii) $C_{\varphi}: \mathcal{B}^{2} \rightarrow \mathcal{B}$ is compact. 
(iii)

$$
\lim _{|\varphi(z)| \rightarrow 1} \frac{1-|z|^{2}}{\left(1-|\varphi(z)|^{2}\right)^{2}}\left|\varphi^{\prime}(z)\right|=0 .
$$

Nor the identity map $\varphi(z)=z$ nor Möbius transformation satisfy the condition (ii) in Theorem 3.1. But there exist inner functions $\varphi$ satisfying the condition (ii) in Theorems 3.1 and 3.2. Such investigation is in [1].

Next we will consider the little Bloch space case and easily obtain the following results by methods similar to the above.

Theorem 3.3. Let $\varphi$ be an analytic self-map of $\mathbb{U}$. Then $C_{\varphi} D: \mathcal{B}_{O} \rightarrow \mathcal{B}_{O}$ is bounded if and only if $C_{\varphi} D: \mathcal{B} \rightarrow \mathcal{B}$ is bounded and $\varphi \in \mathcal{B}_{o}$.

Here we can show the condition $\varphi \in \mathcal{B}_{o}$ by taking $C_{\varphi} D\left(z^{2} / 2\right)=\varphi$, assuming the boundeness of $C_{\varphi} D$ on $\mathcal{B}_{O}$.

Theorem 3.4. Let $\varphi$ be an analytic self-map of $\mathbb{U}$. Then $C_{\varphi} D: \mathcal{B}_{O} \rightarrow \mathcal{B}_{O}$ is compact if and only if

$$
\lim _{|z| \rightarrow 1} \frac{1-|z|^{2}}{\left(1-|\varphi(z)|^{2}\right)^{2}}\left|\varphi^{\prime}(z)\right|=0 .
$$

\section{References}

[1] A. B. Aleksandrov, J. M. Anderson, and A. Nicolau, Inner functions, Bloch spaces and symmetric measures, Proc. London Math. Soc. (3) 79 (1999), no. 2, 318-352.

[2] C. C. Cowen and B. D. MacCluer, Composition Operators on Spaces of Analytic Functions, Studies in Advanced Mathematics. CRC Press, Boca Raton, FL, 1995.

[3] P. L. Duren, Theory of $H^{p}$ Spaces, Academic Press, New York, 1970.

[4] R. A. Hibschweiler and N. Portnoy, Composition followed by differentiation between Bergman and Hardy spaces, Rocky Mountain J. Math. 35 (2005), no. 3, 843-855.

[5] S. Ohno, Products of composition and differentiation between Hardy spaces, Bull. Austral. Math. Soc. 73 (2006), no. 2, 235-243.

[6] S. Ohno, K. Stroehoff, and R. Zhao, Weighted composition operators between Bloch-type spaces, Rocky Mountain J. Math. 33 (2003), no. 1, 191-215.

[7] J. H. Shapiro, Composition Operators and Classical Function Theory, Springer-Verlag, New York, 1993.

[8] W. Smith, Composition operators between Bergman and Hardy spaces, Trans. Amer. Math. Soc. 348 (1996), no. 6, 2331-2348.

[9] K. Zhu, Operator Theory in Function Spaces, Marcel Dekker, New York, 1990.

Nippon Institute of Technology

Miyashiro, Minami-Saitama 345-8501, JAPAN

E-mail address: ohno@nit.ac.jp 